\title{
Prognostic Role of Monocytic Myeloid-Derived Suppressor Cells in Advanced Non-Small-Cell Lung Cancer: Relation to Different Hematologic Indices
}

\author{
Asmaa M. Zahran (iD, ${ }^{1}$ Helal F. Hetta $\left(\mathbb{D},{ }^{2}\right.$ Zeinab Albadry M. Zahran, ${ }^{3}$ Alaa Rashad, ${ }^{4}$ \\ Amal Rayan, ${ }^{5}$ Dalia O. Mohamed, ${ }^{6}$ Zeinab Ahmed Abd Elhameed, ${ }^{3}$ Salah M. Khallaf, ${ }^{7}$ \\ Gaber El-Saber Batiha, ${ }^{8}$ Yasir Waheed ${ }^{D},{ }^{9}$ Khalid Muhammad $\left(D,{ }^{10}\right.$ \\ and Hanaa Nafady-Hego ${ }^{2}$ \\ ${ }^{1}$ Clinical Pathology Department, South Egypt Cancer Institute, Assiut University, Egypt \\ ${ }^{2}$ Department of Medical Microbiology and Immunology, Faculty of Medicine, Assiut University, Assiut 71526, Egypt \\ ${ }^{3}$ Department of Clinical Pathology, Faculty of Medicine, Assiut University, Assiut, Egypt \\ ${ }^{4}$ Department of Chest Diseases and Tuberculosis, Qena Faculty of Medicine, South Valley University, Egypt \\ ${ }^{5}$ Clinical Oncology Department, Faculty of Medicine, Assiut University, Egypt \\ ${ }^{6}$ Radiation Oncology Department, South Egypt Cancer Institute, Assiut University, Egypt \\ ${ }^{7}$ Medical Oncology Department, South Egypt Cancer Institute, Assiut University, Egypt \\ ${ }^{8}$ Department of Pharmacology and Toxicology, Faculty of Veterinary Medicine, Damanhour University, Damanhour City, Egypt \\ ${ }^{9}$ Foundation University Medical College, Foundation University Islamabad, Islamabad 44000, Pakistan \\ ${ }^{10}$ Department of Biology, College of Science, United Arab Emirates University, Al Ain 15551, UAE
}

Correspondence should be addressed to Asmaa M. Zahran; asmaam.zahran@yahoo.com and Khalid Muhammad; k.muhammad@uaeu.ac.ae

Received 25 June 2021; Revised 20 September 2021; Accepted 22 September 2021; Published 11 October 2021

Academic Editor: Sainan Li

Copyright (c) 2021 Asmaa M. Zahran et al. This is an open access article distributed under the Creative Commons Attribution License, which permits unrestricted use, distribution, and reproduction in any medium, provided the original work is properly cited.

Background and Aim. Myeloid-derived suppressor cells (MDSCs) contribute to the process of malignant transformation and tumor progression through immuno- and nonimmunosuppressive mechanisms. The current study is aimed at providing the predictive and prognostic role of Mo-MDSCs in advanced non-small-cell lung cancer (NSCLC) in relation to different hematologic indices. Methods. We recruited 40 cases of advanced NSCLC, stages III and IV, aged $>18-<70$ years old, and eligible to receive chemotherapy with or without radiotherapy, along with 20 healthy controls of comparable age and sex; after diagnosis and staging of patients, blood samples were collected for flow cytometric detection of Mo-MDSCs. Results. Significant accumulation of Mo-MDSCs in patients compared to their controls $(p<0.0001)$. Furthermore, these cells accumulated significantly in stage IV compared to stage III $(p=0.006)$ and correlated negatively with overall survival $(r=-0.471, p=0.002)$, lymphocyte to monocyte ratio $(r=-0.446, p=0.004)$, and mean platelet volume to platelet count ratio $(\mathrm{MPV} / \mathrm{PC})(r=-0.464, p=0.003)$, patients with Mo-MDSCs $<13 \%$ had significantly better survival than those with Mo-MDSCs $\geq 13 \%$ ( $p=0.041)$. Conclusion. Mo-MDSCs represent one of the key mechanisms in the immunosuppressive tumor microenvironment (TME) to play major roles not only in the carcinogenesis of lung cancer but also in disease progression and prognosis and, in addition, predict the efficacy of immune checkpoint inhibitors; our results provided some support to target Mo-MDSCs and needed to be augmented by further studies. 


\section{Introduction}

Globally, lung cancer is one of the leading causes of cancerrelated death [1-3]. Although non-small-cell lung cancer (NSCLC) immunotherapy has fortunately emerged as a relatively promising area of research, immune checkpoint inhibitors have found an influential lantern for NSCLC patients. However, much work remains to elucidate lung tumor immunobiology and how alternative tumor microenvironments (TME) can affect patient survival across different NSCLC subtypes [4].

Recently, studies focused on TME and its role in tumor resistance; tumor suppressor cells within TME, namely, myeloid-derived suppressor cells (MDSCs), greatly attenuate the tumor response to chemotherapy and regrettably immunotherapy and subsequently affect NSCLC prognosis [5].

MDSCs encompass a range of immature cells whose unifying characteristics are their myeloid origin and ability to suppress $\mathrm{T}$ cell activation and $\mathrm{T}$ cell function. Phenotypically, these cells are defined by several markers; none of them is characteristic of MDSCs; the CD11b marker is expressed by all MDSCs [6]; there are two major subtypes of MDSCs; monocytic MDSCs express CD14, and polymorphonuclear MDSCs express CD15 and CD66b; both types express CD33 in addition to CD11b with the absence of HLA-DR.

Growth factors controlling myelopoiesis could induce the accumulation and augment the suppressive activity of MDSCs, including GM-CSF and G-CSF in cancer patients [7]. $\mathrm{C} / \mathrm{EBP} \beta$ transcription factor, which is known to control emergency myelopoiesis, is expressed in chronic inflammation in many solid tumors and different inflammatory conditions, including infection, autoimmunity, obesity, and stress. These conditions have led to the hypothesis that chronic inflammation is a mechanism that increases the risk of cancer and tumor progression by acting as a driving force for MDSCs and subsequently suppressing antitumor immunity [8].

Vascular endothelial growth factor (VEGF), upregulated by hypoxia-inducible factor-1 in hypoxic TME, supports tumor progression through neovascularization. Studies done in NSCLC explored that VEGF attracts MDSCs to the tumor site and further promotes tumor progression [9].

Potent proinflammatory mediators such as IL-6, IL- $1 \beta$, IL-17, and TNF- $\alpha$ accumulate in TME of many cancers to participate in tumor induction and progression; in addition, they induce overexpression and augmented suppressive activity of MDSCs [10-12]; other mediators including prostaglandin E2, cyclooxygenase 2, and proinflammatory calcium-binding proteins S100A8 and S100A9 are implicated in increasing immunosuppressive activity of MDSCs [13, 14].

MDSCs suppress both innate and adaptive immunity through cell to cell contact with their components, with $\mathrm{T}$ cells through sequestering the essential amino acids, cysteine, important for $\mathrm{T}$ cell activation. Furthermore, they downregulate the production of macrophage production of IL-12 favoring the development of tumor-promoting macrophage phenotype. In addition, they inhibit NK-mediated tumor cell lysis and recruit Tregs into the tumor site [15,
16]. Moreover, MDSCs downregulate L-selectin on circulating naïve $\mathrm{T}$ cells, therefore suppressing $\mathrm{T}$ cell activation [17].

Additionally, MDSCs may contribute to carcinogenesis and tumor progression through nonimmunosuppressive mechanisms. Immature myeloid cells directly contribute to skin tumor development by recruiting IL-17-producing CD4+ $\mathrm{T}$ cells [18]. In addition, MDSCs endow stem-like quality to breast cancer cells through IL6/STAT3 and $\mathrm{NO} / \mathrm{NOTCH}$ cross-talk signaling [19]. Moreover, MDSCs could enhance the stemness of cancer cells by inducing microRNA101 and suppressing the corepressor gene Cterminal-binding protein-2 [20].

Huang et al. [21] reported that Mo-MDSCs significantly increased in the peripheral blood of patients with NSCLC compared to healthy controls and correlated with worse prognosis. The current study is aimed at providing the predictive and prognostic role of Mo-MDSCs in advanced NSCLC relating them to different hematologic indices.

\section{Patients and Methods}

This study was a case-controlled study carried out at South Egypt Cancer Institute and Assiut University Hospital and approved by the ethical committee of Assiut University (approval ID no. 17300417). Informed consent was taken from all study participants. The study was conducted in accordance with the Declaration of Helsinki. All experiments were performed in accordance with relevant guidelines and regulations.

We recruited 40 cases of advanced NSCLC, stages III and IV, aged $>18-<70$ years old, and eligible to receive chemotherapy with or without radiotherapy, along with 20 healthy controls of comparable age and sex. Informed consent in written form was taken from all participants. The study objectives were explained to the participants, and then, blood samples were collected by sterilized and safe maneuvers.

We excluded patients with early stages, pretreated patients with chemotherapy, and patients with concurrent excruciating infection.

After diagnosis and staging of patients and before the start of any line of treatment, blood samples were collected for flow cytometric detection of Mo-MDSCs. Systemic chemotherapy was the treatment commonly received in the form of platinum doublets (carboplatin or cisplatin plus either pemetrexed, paclitaxel, gemcitabine, or vinorelbine); some patients especially those with ECOG-PS3 received single agent chemotherapy, while in patients with stage III, concurrent chemoradiation with a 3-dimensional conformal radiotherapy was received after induction chemotherapy.

Lymphocyte to monocyte ratio (LMR) was calculated by dividing the absolute lymphocytic count by the absolute monocytic count of the peripheral blood.

Mean platelet volume to platelet count ratio (MPV/PC) was calculated by dividing the mean platelet volume by total platelet count in $\mu \mathrm{l}$ of peripheral blood.

2.1. Flow Cytometric Detection of Mo-Myeloid Derived Suppressor Cells (Mo-MDSCs). To determine Mo-MDSCs, 


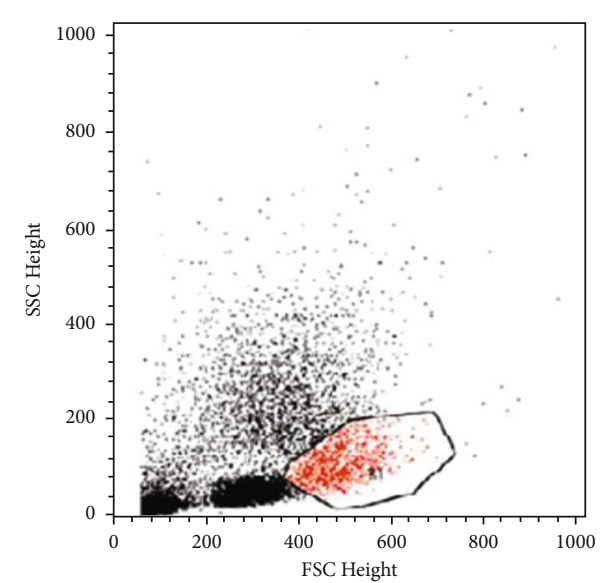

(a)

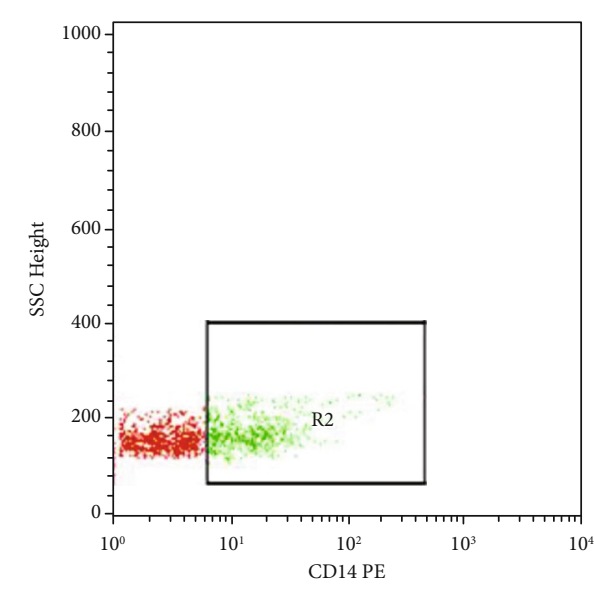

(b)

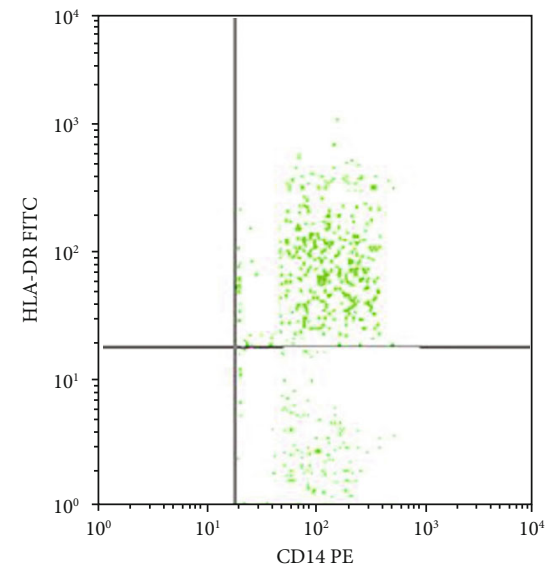

(c)

FIGURE 1: Flow cytometric detection of Mo-myeloid derived suppressor cells (Mo-MDSCs). (a) Forward and side scatter histogram was used to define the monocyte population (R1). (b) The expression of CD14 was assessed in monocytes population (R1) and CD14+ cells were then gated. (c) Then, the expression of HLA-DR on CD14+ cells (R2) was detected, to define CD14+HLA-DR- cells (Mo-MDSCs). Mo-MDSCs were expressed as a percentage of CD14+ cells.

$100 \mu \mathrm{l}$ of blood sample was stained with $10 \mu \mathrm{l}$ of FITCconjugated CD14 and $10 \mu \mathrm{l}$ of Per-CP-conjugated antiHLA-DR; both were purchased from Becton Dickinson Biosciences, USA. Analysis was done by FACSCalibur flow cytometry with Cell Quest software (Becton Dickinson Biosciences, USA). Twenty thousand events were analyzed, and an isotype-matched negative control was used for each sample. The gating strategies to detect the percentage of CD14+HLA-DR- cells (Mo-MDSCs) are shown in Figure 1.

2.2. Statistics. The Shapiro-Wilk test was used to detect normality of our data; all data were normally distributed except percentage monocytes, Mo-MDSCs, absolute monocytic count, absolute lymphocytic count, MPV/PC ratio, and age with $p$ values $<0.001,<0.001,0.002,0.04,0.036$, and 0.021 , respectively, for whom nonparametric tests were applied.

Descriptive statistics including percentages, mean, median, and standard error were used and inferential statistics to determine the significance of data, including independent sample $t$-test, Mann-Whitney $U$ test, Kruskal-Wallis test, and chi-square test. ROC curve was used to find a cutoff value for Mo-MDSCs, Spearman rho correlation was used to determine the degree of association between scale variables, and all data were analyzed using SPSS version 26 and considered significant at $p$ value $<0.05$. Overall survival (OS) was calculated from the time of diagnosis to time of death or last follow-up recorded in patients' files.

\section{Results}

3.1. Accumulation of Mo-MDSCs in NSCLC Patients. At first, our results elucidated a significant accumulation of MoMDSCs in NSCLC patients compared with their comparable healthy controls $(p<0.0001)$ (Table 1$)$.

The mean age of the study patients was 59.5 years with male to female ratio of $1.7: 1$; although smoking was established as a risk factor for lung cancer, $72.5 \%$ of the study patients were either never smokers or past
TABLE 1: Differential accumulation of Mo-MDSCs between NSCLC patients and healthy controls.

\begin{tabular}{lccc}
\hline & $\begin{array}{c}\text { NSCLC } \\
(\text { mean } \pm \text { SE })\end{array}$ & $\begin{array}{c}\text { Controls } \\
(\text { mean } \pm \text { SE })\end{array}$ & $p$ value \\
\hline Mo-MDSCs & $13.99 \pm 0.55$ & $4.15 \pm 0.2$ & $<0.0001$ \\
Absolute monocytic count & $12.29 \pm 0.32$ & $7.41 \pm 0.29$ & $<0.0001$ \\
\hline
\end{tabular}

Data expressed as mean \pm SE and analyzed by Mann-Whitney $U$ test.

smokers. ECOG-PS is one of the two most commonly used performance scales; considering that NSCLC is a debilitating disease and commonly manifested at a later stage, poor performance status was evident in our study ( $70 \%$ of the patients had ECOG-PS $>1$ ); adenocarcinoma was the commonest pathologic type expressed in $57.5 \%$ of the patients, and as expected, stage IV was evident in $42.5 \%$ of the patients; the rest of the characteristics are illustrated in Table 2.

As expected, the significant accumulation of Mo-MDSCs in stage IV than stage III confirms the possible role of these cells in disease progression and indirectly referred to the role of immune-mediated destruction of tumor cells; however, these cells did not exhibit any significant change with other clinical characteristics (Table 3).

3.2. Correlations between Mo-MDSCs and Overall Survival. The mean Mo-MDSC percentage expressed negative correlations with OS, MPV/PC ratio, LMR, and with the number of cycles of chemotherapy received (Table 4); further analysis demonstrated that a negative correlation between OS and Mo-MDSCs was clearly apparent in males but not in females (Figure 2).

Eight out of nine, 10/14, and 1/17 patients with stages IIIA, IIIB, and IV, respectively, achieved more than one-year OS compared to $1 / 9,4 / 14$, and $16 / 17$ patients of the previous stages that had lower than one-year OS and the results were significant $(p<0.0001)$; additionally, the mean percentage of Mo-MDSCs for those with more than one-year survival was 
TABLE 2: Clinicopathologic characteristics of 40 patients with NSCLC.

\begin{tabular}{lc}
\hline Characteristics & Descriptive \\
\hline Age (mean \pm SE) & $58.5 \pm 1.7$ \\
Median & $59.5 \mathrm{y}$ \\
Sex (male/female) & $25 / 15$ \\
Smoking & \\
Never smoker & $17(42.5 \%)$ \\
Current smoker & $11(27.5 \%)$ \\
Past smoker & $12(30 \%)$ \\
ECOG-PS & \\
PS $=1$ & $12(30 \%)$ \\
PS $=2$ & $13(32.5 \%)$ \\
PS $=3$ & $15(37.5 \%)$ \\
Histopathology & \\
Squamous cell carcinoma & $12(30 \%)$ \\
Adenocarcinoma & $23(57.5 \%)$ \\
Large cell carcinoma & $4(10 \%)$ \\
Bronchoalveolar carcinoma & $1(2.5 \%)$ \\
Stage & \\
IIIA & $9(22.5 \%)$ \\
IIIB & $14(35 \%)$ \\
IV & $17(42.5 \%)$ \\
LMR (mean \pm SE) & $3($ range: $0-7)$ \\
MPV/PC ratio (mean \pm SE) & $2.62 \pm 0.23$ \\
Relative monocytic count & $0.36 \pm 0.02$ \\
Relative lymphocytic count & $12.29 \pm 0.22$ \\
Outcome & $20.0 \pm 1.94$ \\
Dead & \\
Alive & $26(65 \%)$ \\
\hline L & $14(35 \%)$ \\
\hline &
\end{tabular}

LMR: lymphocyte to monocyte ratio; MPV/PC ratio: mean platelet volume to platelet count ratio; ECOG-PS: Eastern Cooperative Oncology Groupperformance status.

13.01 compared with 14.79 for those with lower than one-year survival $(p=0.021$, Figure 3$)$.

3.3. Overall Survival. The ROC curve was performed to find a cutoff point of Mo-MDSCs \% at which the overall survival significantly differed, and it was $\approx 13 \%$ with $\mathrm{AUC}=0.742 \pm$ $0.1 \quad(95 \% \mathrm{CI}=0.59-0.9, p=0.009)$; OS was significantly higher for those with Mo-MDSCs $<13 \%$ than those with Mo-MDSCs $\geq 13 \%$ (17.64 vs. $11.0, p=0.022) \quad$ (Table 5, Figure 4).

The mean \pm SD for LMR and MPV/PC ratio for patients with $>12$ months of survival were $3.63 \pm 0.92$ and $0.44 \pm 0.1$ compared to $1.7 \pm 1.3$ and $0.29 \pm 0.1$ for those with $<12$ months of survival ( $p<0.0001$ and $p<0.0001$, respectively) (Figure 5).

Furthermore, the distribution of MPV/PC and LMR was significantly different according to the cutoff point value of Mo-MDSCs, where they significantly accumulated in those
TABLE 3: Relation of Mo-MDSCs to different clinical characteristics.

\begin{tabular}{lcc}
\hline Characteristics & Mo-MDSCs $($ mean \pm SE) & $p$ value \\
\hline Sex & & 0.1 \\
Male & $14.43 \pm 0.51$ & \\
Female & $13.13 \pm 0.57$ & \\
Smoking & & 0.2 \\
Never smoker & $13.13 \pm 0.5$ & \\
Current smoker & $14.93 \pm 0.72$ & \\
Past smoker & $14.2 \pm 0.83$ & \\
ECOG-PS & & 0.09 \\
PS $=1$ & $13.38 \pm 0.54$ & \\
PS $=2$ & $13.21 \pm 0.73$ & \\
PS $=3$ & $15.04 \pm 0.66$ &
\end{tabular}

Histopathology

0.8

Squamous cell carcinoma

$13.74 \pm 0.83$

Adenocarcinoma

$14.0 \pm 0.49$

Large cell carcinoma

$13.0 \pm 0.0$

Bronchoalveolar carcinoma

$15.2 \pm 1.3$

Stage

0.0.003*

IIIA $\quad 12.51 \pm 0.4$

IIIB $\quad 13.2 \pm 0.61$

IV $\quad 15.32 \pm 0.61$

Data analyzed by Mann-Whitney $U$ test and Kruskal-Wallis test. $r$ : Spearman coefficient; * significant.

with Mo-MDSCs $<13 \%$ compared to those with $\geq 13 \%$ (for $\mathrm{MPC} / \mathrm{PV}, 0.4 \pm 0.1$ vs. $0.3 \pm 0.1, p=0.008$; for $\mathrm{LMR}, 3.4 \pm$ 1.11 vs. $2.2 \pm 1.5, p=0.005$ ) (Figures $6(\mathrm{a})$ and $6(\mathrm{~b})$ ).

3.4. Multiple Linear Regression Test for Different Predictors of OS. Multiple linear regression was run to predict OS from 8 predictors found to significantly affect the mean OS including age, sex, smoking, stage, performance status, Mo-MDSCs, MPV/PC ratio, and LMR; these prementioned variables collectively predicted $\mathrm{OS}$ with significant impact $\left(F(8,31)=14.230, p<0.0001, R^{2}=0.786\right)$; however, only stage and LMR added significantly to the prediction of OS. Looking at Mo-MDSCs, $B=0.018$ and $p=0.9$ denoted that only $1.8 \%$ of the change in OS variance was attributed to Mo-MDSC change when all remaining predictors were held constant. Furthermore, it was misleading as for each one of the percentage increase in Mo-MDSCs, there was an increase in OS by 0.018 months; in addition, it was not significant (Table 6).

\section{Discussion}

Nowadays, it is well established that the tumor microenvironment and immune system play a crucial role in the initiation and progression of different cancers, including NSCLC [22]. MDSCs are considered the major suppressor of the immune system interfering with both innate and adaptive 
TABLE 4: Correlation between Mo-MDSCs and survival.

\begin{tabular}{|c|c|c|c|c|c|c|c|}
\hline & & Mo-MDSCs & Age & $\mathrm{MPV} / \mathrm{PC}$ ratio & Number of cycles & OS in months & LMR \\
\hline \multirow{2}{*}{ Mo-MDSCs } & $r$ & - & 0.016 & -0.464 & -0.412 & -0.471 & -0.446 \\
\hline & $p$ & - & 0.920 & 0.003 & 0.008 & 0.002 & 0.004 \\
\hline \multirow{2}{*}{ Age } & $r$ & 0.016 & - & -0.215 & 0.014 & -0.248 & -0.209 \\
\hline & $p$ & 0.920 & & 0.183 & 0.931 & 0.123 & 0.195 \\
\hline \multirow{2}{*}{$\mathrm{MPV} / \mathrm{PC}$ ratio } & $r$ & -0.464 & -0.215 & - & -0.010 & 0.737 & 0.548 \\
\hline & $p$ & 0.003 & 0.183 & - & 0.951 & $<0.0001$ & $<0.0001$ \\
\hline \multirow{2}{*}{ Number of cycles } & $r$ & -0.412 & 0.014 & -0.010 & - & 0.036 & 0.285 \\
\hline & $p$ & 0.008 & 0.931 & 0.951 & - & 0.826 & 0.075 \\
\hline \multirow{2}{*}{ OS in months } & $r$ & -0.471 & -0.248 & 0.737 & 0.036 & - & 0.718 \\
\hline & $p$ & 0.002 & 0.123 & 0.0001 & 0.826 & - & $<0.0001$ \\
\hline \multirow{2}{*}{ LMR } & $r$ & -0.446 & -0.209 & 0.548 & 0.285 & 0.718 & - \\
\hline & $p$ & 0.004 & 0.195 & 0.0001 & 0.075 & $<0.0001$ & - \\
\hline
\end{tabular}

Data analyzed by Spearman rho correlation. $r$ : correlation coefficient.

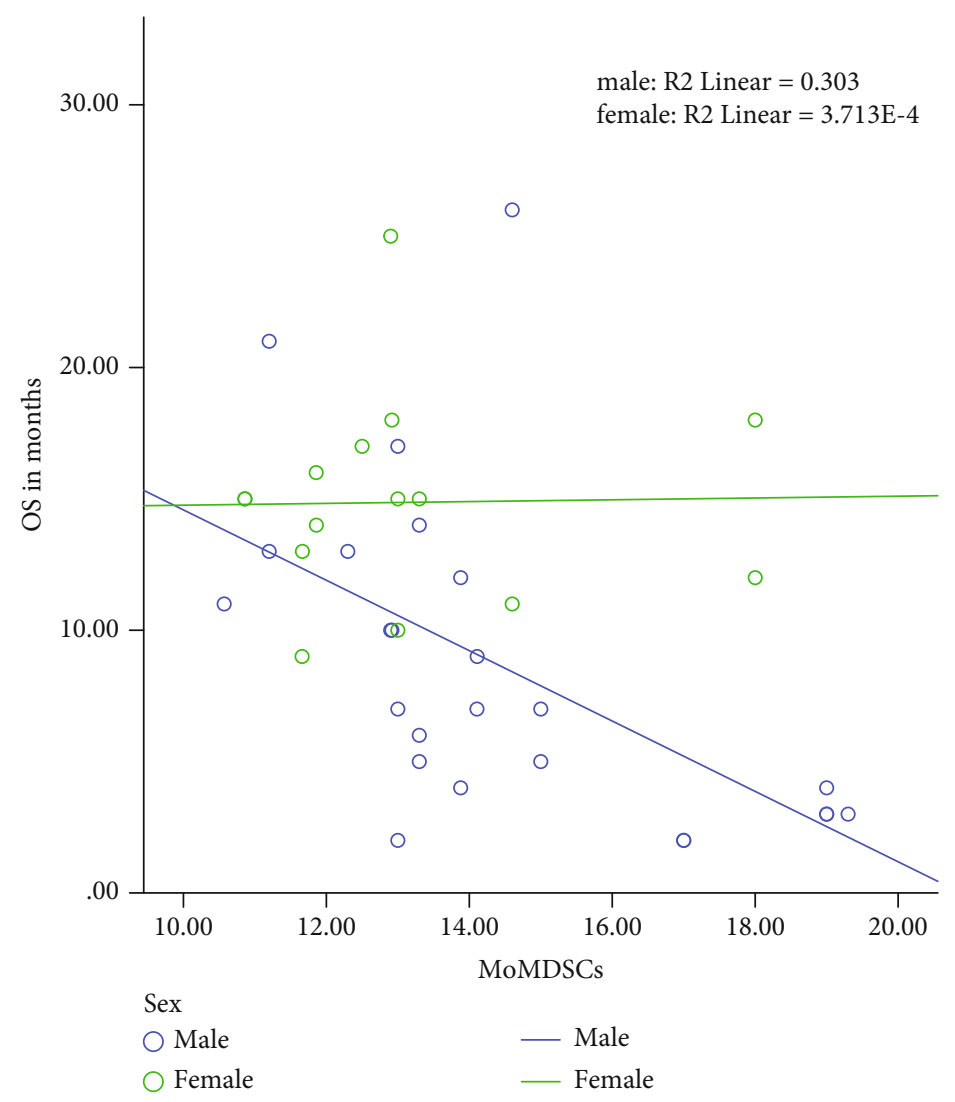

FIGURE 2: Correlation between overall survival (OS) and myeloid-derived suppressor cells (MDSCs) according to sex.

immune responses. Mo-MDSCs were detected to be highly expressed in peripheral blood than excised tissues and lymph nodes of NSCLC patients [23]; subsequently, flow cytometric analysis of peripheral blood for detection of these cells is a reliable method.

In the current study, Mo-MDSCs were significantly more prevalent in the peripheral blood of NSCLC patients than healthy controls. Furthermore, increased levels of these cells were associated with poor prognostic features, including advanced stage, low LMR, low MPV/PC ratio, and poor overall survival.

Mo-MSDCs were reported to produce TGF- $\beta$ in the peripheral blood [24]; furthermore, TGF- $\beta$ was known to induce immunosuppression and promote angiogenesis in TME; in another study, this cytokine was produced by Mo-MDSCs in all tissues [23]. 


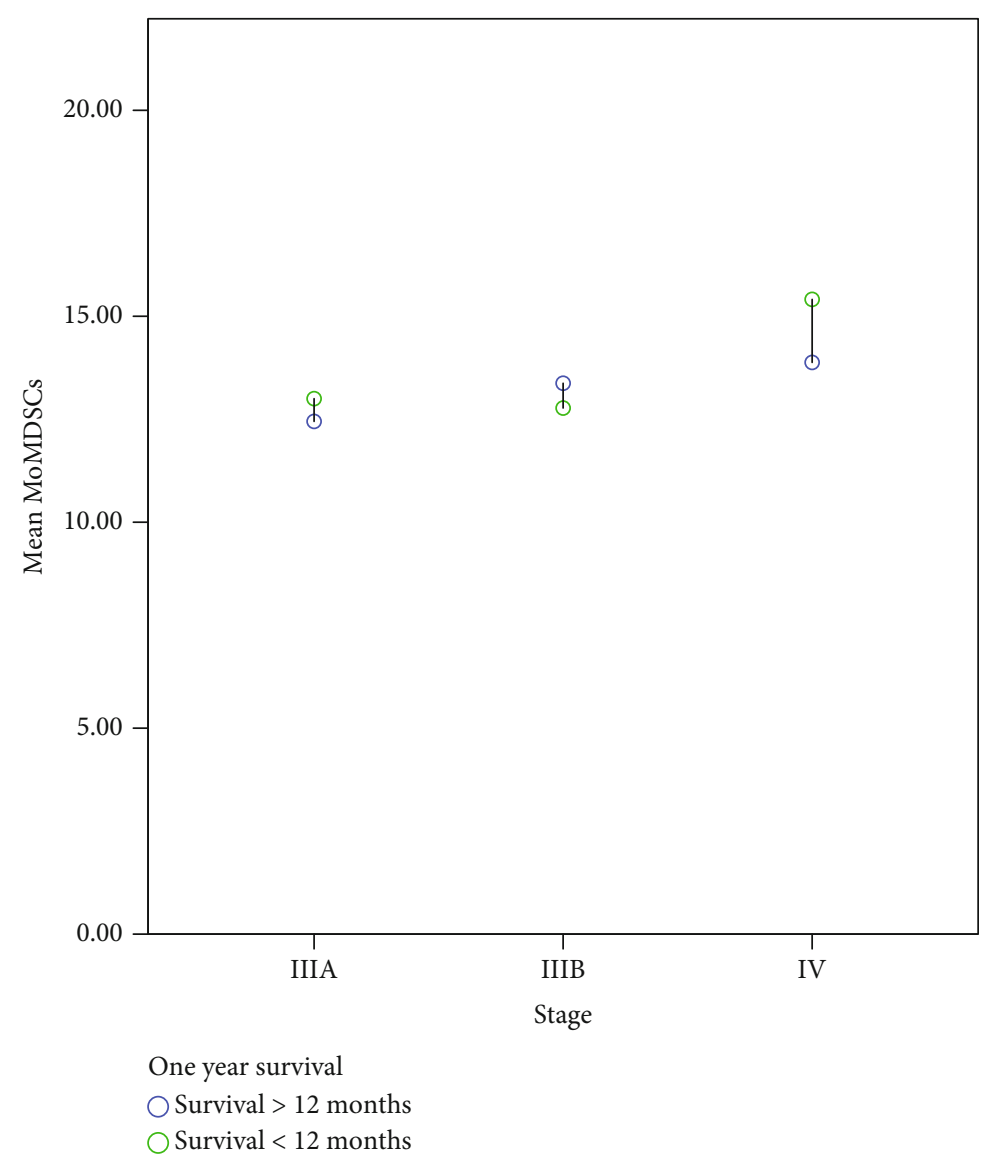

FIgURE 3: Differences in the mean myeloid-derived suppressor cells (Mo-MDSCs) according to 1-year survival in each stage, $p<0.0001$.

TABLE 5: Difference of OS according to Mo-MDSC levels among NSCLC patients.

\begin{tabular}{lcc}
\hline OS & Mo-MDSCs $<13 \%$ & Mo-MDSCs $\geq 13 \%$ \\
\hline Mean \pm SE & $17.64 \pm 1.51$ & $11.0 \pm 1.6$ \\
$95 \%$ CI & $14.7-20.6$ & $7.9-14.04$ \\
Logrank, $p$ value & \multicolumn{2}{c}{$5.21, p=0.022$} \\
\hline
\end{tabular}

Data analyzed by Kaplan-Meier with logrank for comparison.

Several studies demonstrated that NSCLC was associated with high levels of Mo-MDSCs which in turn were responsible for the resistance of this tumor to different systemic therapies [21, 25-27].

Yamauchi et al. [28] showed that a significant increase in the percentage of circulating Mo-MDSCs was observed in patients with resectable non-small-cell lung cancer compared with healthy donors; in addition, preoperative levels of MoMDSCs predicted recurrence-free survival after surgery.

A meta-analysis of 40 studies supported the existence of an association between higher MDSC levels and worse clinical outcomes in solid malignancies including NSCLC where total MDSCs $(\mathrm{HR}=3.386 ; 95 \% \mathrm{CI}=1.618-7.087)$, M-MDSCs $(\mathrm{HR}=2.834 ; 95 \% \mathrm{CI}=2.128-3.774)$, and PMNMDSCs $(\mathrm{HR}=1.915 ; 95 \% \mathrm{CI}=1.420-2.583)$ were all associ- ated with worse disease-free survival, progression-free survival, and recurrence-free survival [29].

In accordance with the previously mentioned studies, our results depicted significantly increased levels of MoMDSCs in patients compared with healthy controls in addition to an association of these cells with poor survival in NSCLC patients.

Interestingly, immunophenotyping analysis was performed on peripheral blood samples from seven patients with lung cancer unfit for surgery and treated with stereotactic body radiotherapy (SBRT) to evaluate the impact of SBRT on patients' immune cells including Mo-MDSCs and reported a significant decrease in these cells after RT [30] implicating that not only chemotherapy affected the levels of immune system; however, there was no uniform effect of chemotherapy on the peripheral blood percentages or immunosuppressive function of Mo-MDSCs, but three cycles of bevacizumabbased chemotherapy were associated with significantly reduced level of these cells [31]. Our study agreed with the previous one, where the percentage of Mo-MDSCs negatively correlated with the number of cycles of chemotherapy.

The percentage of MDSCs in patients with colorectal cancer with $\mathrm{LMR} \leq 2.4$ was statistically higher than that with LMR $>2.4 \quad(p=0.012)$. Those patients with $\mathrm{LMR} \leq 2.4$ exhibited a statistically lower RFS than those with LMR > $2.4(p=0.008)[32]$. Likewise, a negative correlation between 


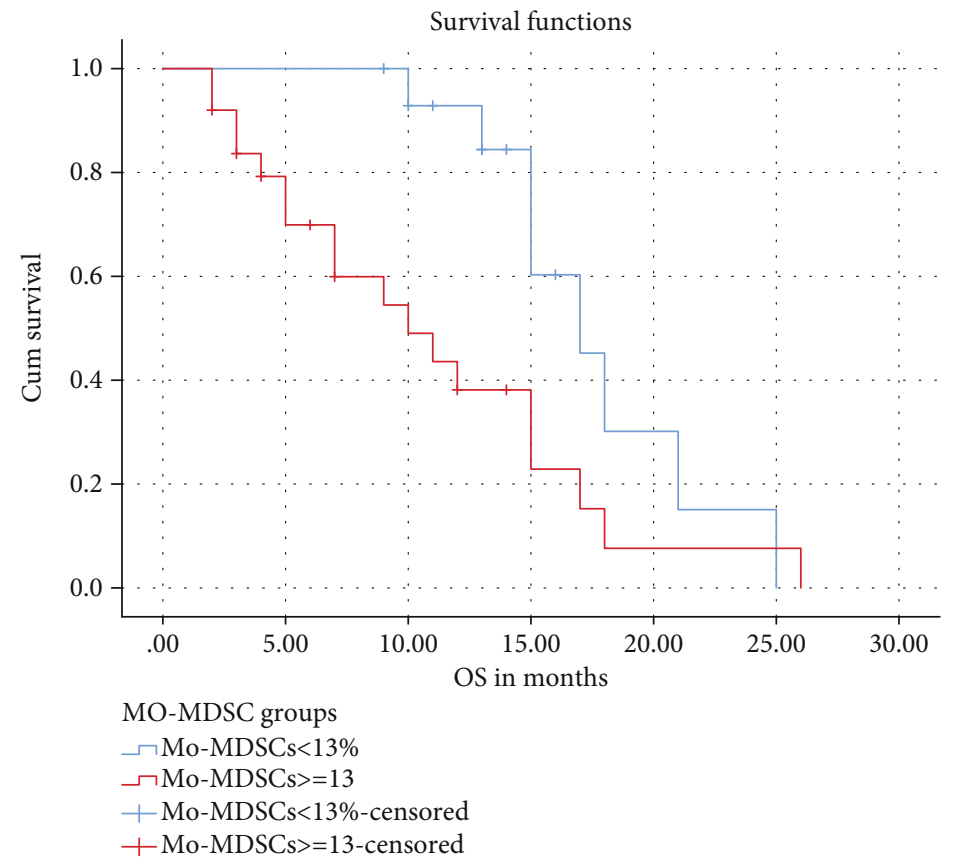

FIGURE 4: Differences in overall survival (OS) according to myeloid-derived suppressor cell (Mo-MDSC) cutoff value.

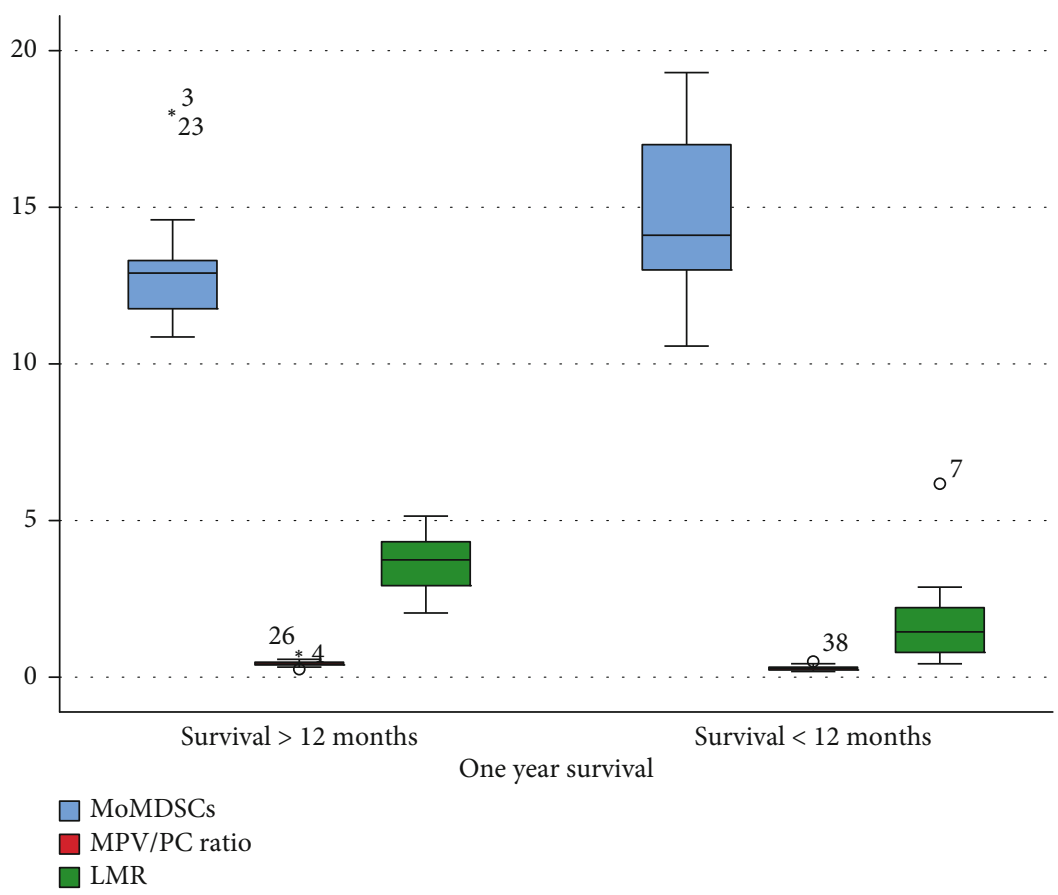

FIGURE 5: Differences in myeloid-derived suppressor cells (Mo-MDSCs), lymphocyte to monocyte ratio (LMR), and mean platelet volume to platelet count (MPV/PC) ratio for patients with $>12$ months of survival compared to patients $<12$ months of survival, data analyzed by Mann-Whitney test and independent sample $t$-test.

LMR and Mo-MDSCs was evident in our work with significant effect $(r=-0.446, p=0.004)$.

Wang et al. proved that low pretreatment LMR was with poor OS $(\mathrm{HR}=1.63,95 \% \mathrm{CI}: 1.44-1.85, p<0.001)$ and PFS (HR $=1.49,95 \% \mathrm{CI}: 1.25-1.77, p<0.001)$ compared to those NSCLC patients with high LMR in their meta- analysis of 20 articles discussing the role of LMR in NSCLC [33].

$\mathrm{MPV} / \mathrm{PC}$ was established as a prognostic factor in both univariate and multivariate analysis of NSCLC with a cutoff value of 0.408730 , where low MPV/PC ratio was associated with poor OS (hazard ratio (HR): $1.668,95 \% \mathrm{CI}: 1.235-$ 


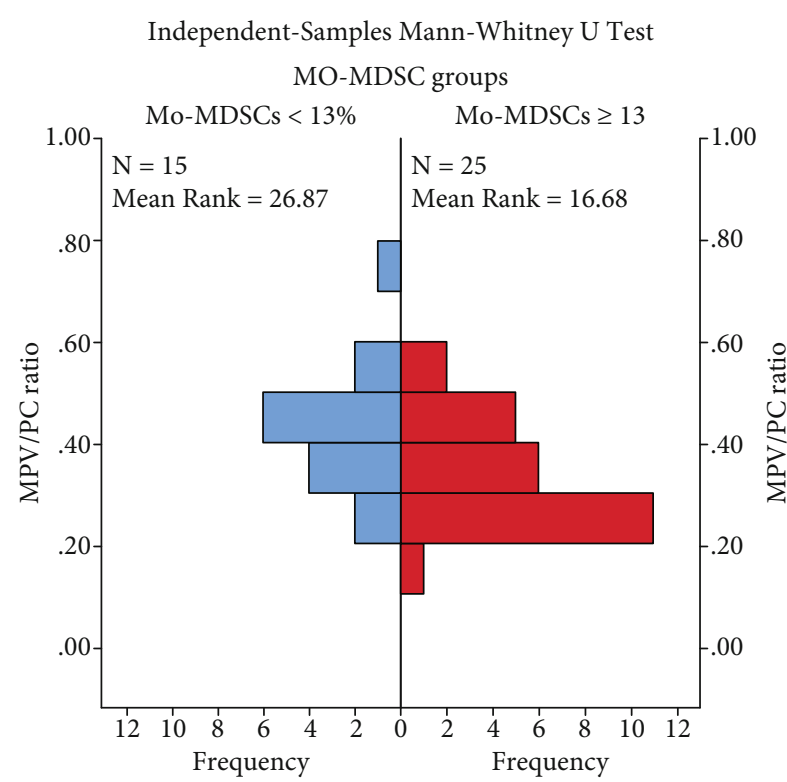

(a)

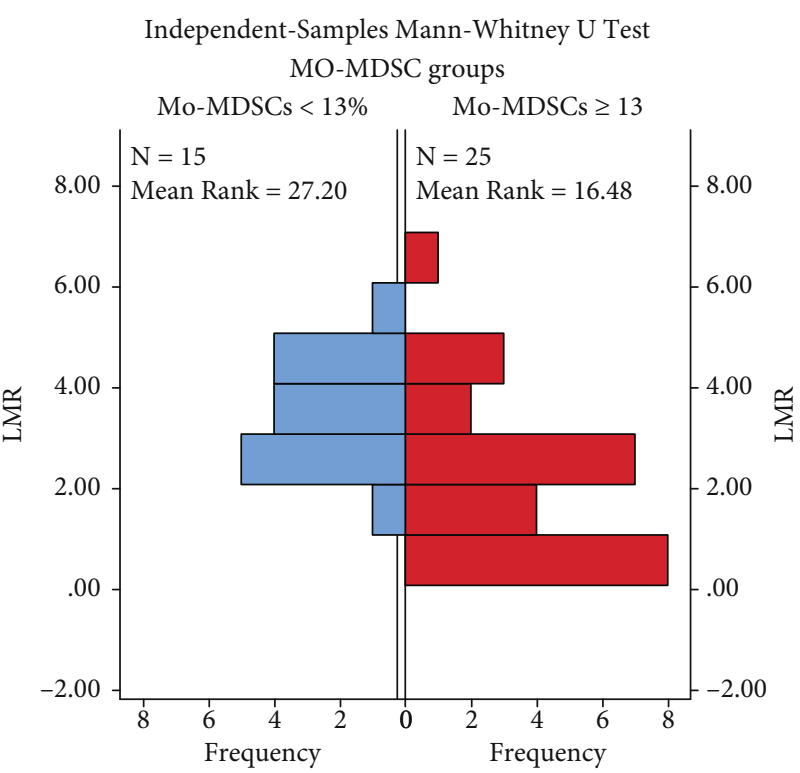

(b)

Figure 6: (a) Differences of MPV/PC according to cutoff point values of Mo-MDSCs. (b) Differences of LMR according to cutoff point values of Mo-MDSCs.

TABLE 6: Multiple linear regression test of different predictors of OS.

\begin{tabular}{|c|c|c|c|c|c|c|}
\hline & \multicolumn{2}{|c|}{$\begin{array}{l}\text { Unstandardized } \\
\text { coefficients }\end{array}$} & \multirow{2}{*}{$t$} & \multirow{2}{*}{ Sig. } & \multicolumn{2}{|c|}{$95 \%$ confidence interval for $B$} \\
\hline & $B$ & Std. error & & & Lower bound & Upper bound \\
\hline (Constant) & 6.817 & 7.540 & 0.904 & 0.373 & -8.561 & 22.196 \\
\hline Mo-MDSCs & 0.018 & 0.252 & 0.073 & 0.943 & -0.496 & 0.533 \\
\hline Smoking & 0.804 & 1.102 & 0.730 & 0.471 & -1.444 & 3.052 \\
\hline $\mathrm{MPV} / \mathrm{PC}$ ratio & 12.246 & 6.465 & 1.894 & 0.068 & -0.939 & 25.431 \\
\hline ECOG-PS & -1.141 & 0.887 & -1.286 & 0.208 & -2.950 & 0.668 \\
\hline Sex & 1.739 & 2.046 & 0.850 & 0.402 & -2.435 & 5.913 \\
\hline Stage & -2.926 & 1.033 & -2.832 & 0.008 & -5.033 & -0.819 \\
\hline LMR & 1.295 & 0.506 & 2.561 & 0.016 & 0.264 & 2.326 \\
\hline Age & 0.017 & 0.063 & 0.279 & 0.782 & -0.111 & 0.146 \\
\hline
\end{tabular}

Data analyzed by linear regression with enter method.

2.271, $p=0.0008$ ) [34]; also, low MPV/PC ratio was associated with poor prognostic features including advanced stage and poor performance status in these patients [35]. Our results in turn adhered to the previous studies where LMR and MPV/PC ratio were negatively correlated with OS.

The inverse relation between MDSCs and LMR remains to be elucidated; increased peripheral MDSCs contribute to peripheral monocytosis and hence lower LMR; in addition, it is established that peripheral monocytosis has been reported to be associated with poor clinical outcomes [32]; we reported significant monocytosis in NSCLC patients compared to their controls with negative correlation between LMR and Mo-MDSCs in our patients.

It is worth mentioning that limited data are available to adequately enforce our finding regarding the negative correlation between Mo-MDSCs and MPV/PC ratio; however, in line with previous studies relating $\mathrm{MPV} / \mathrm{PC}$ ratio to poor prognosis in NSCLC, subsequently, a low MPV/PC ratio may be correlated with increased Mo-MDSCs.

Several limitations exist in the current study, including the small number of enrolled patients, immunohistochemical evaluation of MDSCs in tumor tissues not done, and heterogeneity of the studied patients. Future work is recommended including addition of CD14, CD11b, CD15, and CD66b immunohistochemistry and qPCR of tissue to verify the hypothesis further. Also, data about the proinflammatory cytokines including IL6, IL8, and TNF $\alpha$ are needed to demonstrate the relationship between inflammation factors and MDSCs.

In conclusion, Mo-MDSCs represent one of the key mechanisms in immunosuppressive TME to play major roles not only in the carcinogenesis of lung cancer but also in disease progression and prognosis and, in addition, predict the efficacy of immune checkpoint inhibitors; our results 
provided some support to target Mo-MDSCs and needed to be augmented by further studies.

\section{Data Availability}

The data used to support the findings of this study are available from the corresponding authors upon request.

\section{Conflicts of Interest}

The authors declare that they have no conflicts of interest.

\section{Authors' Contributions}

All authors contributed in research design, carrying out the experiments, data analysis, and writing the manuscript.

\section{References}

[1] F. Bray, J. Ferlay, I. Soerjomataram, R. L. Siegel, L. A. Torre, and A. Jemal, "Global cancer statistics 2018: GLOBOCAN estimates of incidence and mortality worldwide for 36 cancers in 185 countries," CA: a Cancer Journal for Clinicians, vol. 68, no. 6, pp. 394-424, 2018.

[2] H. F. Hetta, A. M. Zahran, R. I. el-Mahdy et al., "Assessment of circulating miRNA-17 and miRNA-222 expression profiles as non-invasive biomarkers in Egyptian patients with nonsmall-cell lung cancer," Asian Pacific Journal of Cancer Prevention, vol. 20, no. 6, pp. 1927-1933, 2019.

[3] H. F. Hetta, A. M. Zahran, E. A. Shafik et al., "Circulating miRNA-21 and miRNA-23a expression signature as potential biomarkers for early detection of non-small-cell lung cancer," Microrna, vol. 8, no. 3, pp. 206-215, 2019.

[4] M. Aldarouish and C. Wang, "Trends and advances in tumor immunology and lung cancer immunotherapy," Journal of Experimental \& Clinical Cancer Research, vol. 35, no. 1, p. 157, 2016.

[5] M. Tavakkoli, C. R. Wilkins, J. V. Mones, and M. J. Mauro, “A novel paradigm between leukocytosis, G-CSF secretion, neutrophil-to-lymphocyte ratio, myeloid-derived suppressor cells, and prognosis in non-small cell lung cancer," Frontiers in Oncology, vol. 9, p. 295, 2019.

[6] S. Ostrand-Rosenberg and C. Fenselau, "Myeloid-derived suppressor cells: immune-suppressive cells that impair antitumor immunity and are sculpted by their environment," The Journal of Immunology, vol. 200, no. 2, pp. 422-431, 2018.

[7] P. Filipazzi, R. Valenti, V. Huber et al., "Identification of a new subset of myeloid suppressor cells in peripheral blood of melanoma patients with modulation by a granulocytemacrophage colony-stimulation factor-based antitumor vaccine," Journal of Clinical Oncology, vol. 25, no. 18, pp. 2546-2553, 2007.

[8] S. Ostrand-Rosenberg and P. Sinha, "Myeloid-derived suppressor cells: linking inflammation and cancer," The Journal of Immunology, vol. 182, no. 8, pp. 4499-4506, 2009.

[9] S. Kusmartsev, E. Eruslanov, H. Kübler et al., "Oxidative stress regulates expression of VEGFR1 in myeloid cells: link to tumor-induced immune suppression in renal cell carcinoma," The Journal of Immunology, vol. 181, no. 1, pp. 346-353, 2008.

[10] S. K. Bunt, L. Yang, P. Sinha, V. K. Clements, J. Leips, and S. Ostrand-Rosenberg, "Reduced inflammation in the tumor microenvironment delays the accumulation of myeloidderived suppressor cells and limits tumor progression," Cancer Research, vol. 67, no. 20, pp. 10019-10026, 2007.

[11] X. Song, Y. Krelin, T. Dvorkin et al., "CD11b+/Gr-1+ immature myeloid cells mediate suppression of $\mathrm{T}$ cells in mice bearing tumors of IL- $1 \beta$-secreting cells," The Journal of Immunology, vol. 175, no. 12, pp. 8200-8208, 2005.

[12] S. Tu, G. Bhagat, G. Cui et al., "Overexpression of interleukin$1 \beta$ induces gastric inflammation and cancer and mobilizes myeloid-derived suppressor cells in mice," Cancer Cell, vol. 14, no. 5, pp. 408-419, 2008.

[13] W. Choksawangkarn, L. M. Graham, M. Burke et al., "Peptidebased systems analysis of inflammation induced myeloidderived suppressor cells reveals diverse signaling pathways," Proteomics, vol. 16, no. 13, pp. 1881-1888, 2016.

[14] Y. Mao, I. Poschke, E. Wennerberg et al., "Melanoma-educated CD14+ cells acquire a myeloid-derived suppressor cell phenotype through COX-2-dependent mechanisms," Cancer Research, vol. 73, no. 13, pp. 3877-3887, 2013.

[15] S. Ostrand-Rosenberg, "Myeloid-derived suppressor cells: more mechanisms for inhibiting antitumor immunity," Cancer Immunology, Immunotherapy, vol. 59, no. 10, pp. 1593$1600,2010$.

[16] M. Elkabets, V. S. Ribeiro, C. A. Dinarello et al., "IL-1 $\beta$ regulates a novel myeloid-derived suppressor cell subset that impairs NK cell development and function," European Journal of Immunology, vol. 40, no. 12, pp. 3347-3357, 2010.

[17] E. M. Hanson, V. K. Clements, P. Sinha, D. Ilkovitch, and S. Ostrand-Rosenberg, "Myeloid-derived suppressor cells down-regulate L-selectin expression on CD4+ and CD8+ T cells," The Journal of Immunology, vol. 183, no. 2, pp. 937-944, 2009.

[18] M. L. Ortiz, V. Kumar, A. Martner et al., "Immature myeloid cells directly contribute to skin tumor development by recruiting IL-17-producing CD4+ T cells," Journal of Experimental Medicine, vol. 212, no. 3, pp. 351-367, 2015.

[19] D. Peng, T. Tanikawa, W. Li et al., "Myeloid-derived suppressor cells endow stem-like qualities to breast cancer cells through IL6/STAT3 and NO/NOTCH cross-talk signaling," Cancer Research, vol. 76, no. 11, pp. 3156-3165, 2016.

[20] T. X. Cui, I. Kryczek, L. Zhao et al., "Myeloid-derived suppressor cells enhance stemness of cancer cells by inducing microRNA101 and suppressing the corepressor CtBP2," Immunity, vol. 39, no. 3, pp. 611-621, 2013.

[21] A. Huang, B. Zhang, B. Wang, F. Zhang, K. X. Fan, and Y. J. Guo, "Increased CD14(+)HLA-DR (-/low) myeloid-derived suppressor cells correlate with extrathoracic metastasis and poor response to chemotherapy in non-small cell lung cancer patients," Cancer Immunology, Immunotherapy, vol. 62, no. 9, pp. 1439-1451, 2013.

[22] A. M. Zahran, H. F. Hetta, S. Mansour, E. S. Saad, and A. Rayan, "Reviving up dendritic cells can run cancer immune wheel in non-small cell lung cancer: a prospective two-arm study," Immunotherapy, vol. 70, no. 3, pp. 733-742, 2021.

[23] K. Pogoda, M. Pyszniak, P. Rybojad, and J. Tabarkiewicz, "Monocytic myeloid-derived suppressor cells as a potent suppressor of tumor immunity in non-small cell lung cancer," Oncology Letters, vol. 12, no. 6, pp. 4785-4794, 2016.

[24] B. De Keersmaecker, K. Fostier, J. Corthals et al., "Immunomodulatory drugs improve the immune environment for dendritic cell-based immunotherapy in multiple myeloma patients 
after autologous stem cell transplantation," Cancer Immunology, Immunotherapy, vol. 63, no. 10, pp. 1023-1036, 2014.

[25] C. Y. Liu, Y. M. Wang, C. L. Wang et al., "Population alterations of L-arginase- and inducible nitric oxide synthaseexpressed CD11b+/CD14/CD15+/CD33+ myeloid-derived suppressor cells and CD8+ T lymphocytes in patients with advanced-stage non-small cell lung cancer," Journal of Cancer Research and Clinical Oncology, vol. 136, no. 1, pp. 35-45, 2010.

[26] Q. Zhou, X. Tang, X. Tian et al., "LncRNA MALAT1 negatively regulates MDSCs in patients with lung cancer," Journal of Cancer, vol. 9, no. 14, pp. 2436-2442, 2018.

[27] E. K. Vetsika, F. Koinis, M. Gioulbasani et al., "A circulating subpopulation of monocytic myeloid-derived suppressor cells as an independent prognostic/predictive factor in untreated non-small lung cancer patients," Journal of Immunology Research, vol. 2014, Article ID 659294, 12 pages, 2014.

[28] Y. Yamauchi, S. Safi, C. Blattner et al., "Circulating and tumor myeloid-derived suppressor cells in resectable non-small cell lung cancer," American Journal of Respiratory and Critical Care Medicine, vol. 198, no. 6, pp. 777-787, 2018.

[29] P.-F. Wang, S.-Y. Song, T.-J. Wang et al., "Prognostic role of pretreatment circulating MDSCs in patients with solid malignancies: a meta-analysis of 40 studies," OncoImmunology, vol. 7, no. 10, article e1494113, 2018.

[30] A. Navarro-Martín, I. L. Galiana, M. A. Berenguer Frances et al., "Preliminary study of the effect of stereotactic body radiotherapy (SBRT) on the immune system in lung cancer patients unfit for surgery: immunophenotyping analysis," International Journal of Molecular Sciences, vol. 19, no. 12, p. 3963, 2018.

[31] F. Koinis, E. K. Vetsika, D. Aggouraki et al., "Effect of first-line treatment on myeloid-derived suppressor cells' subpopulations in the peripheral blood of patients with non-small cell lung cancer," Journal of Thoracic Oncology, vol. 11, no. 8, pp. 1263-1272, 2016.

[32] T. Shimura, M. Shibata, K. Gonda et al., "Prognostic impact of preoperative lymphocyte-to-monocyte ratio in patients with colorectal cancer with special reference to myeloid-derived suppressor cells," Fukushima Journal of Medical Science, vol. 64 , no. 2 , pp. $64-72,2018$.

[33] Y. Wang, D. Huang, W. Y. Xu, Y. W. Wang, and G. W. Che, "Prognostic value of pretreatment lymphocyte-to-monocyte ratio in non-small cell lung cancer: a meta-analysis," Oncology Research and Treatment, vol. 42, no. 10, pp. 523-531, 2019.

[34] N. Inagaki, K. Kibata, T. Tamaki, T. Shimizu, and S. Nomura, "Prognostic impact of the mean platelet volume/platelet count ratio in terms of survival in advanced non-small cell lung cancer," Lung Cancer, vol. 83, no. 1, pp. 97-101, 2014.

[35] A. Ibrahim and O. Abdel Fattah, "The relation between mean platelet volume/platelet count ratio and prognostic factors in patients with advanced non-small cell lung cancer," Research in Oncology, vol. 12, no. 1, pp. 15-19, 2016. 